\title{
FACTORES DE RIESGO DE ENFERMEDAD RENAL CRÓNICA
}

\author{
Roberto D'Achiardi Rey M.D, FACP, JuAn Guillermo Vargas M.D, MACP, \\ Jorge EnriQue EcheVerri M.D, Mauricio Moreno M.D, Gustavo Quiroz M.D.
}

\section{Resumen}

La enfermedad renal crónica es una patología muy frecuente asociado con múltiples coomorbilidades. La fisiopatología del daño endotelial es diferente en los pacientes con enfermedad renal crónica y en este es un campo de investigación en la actualidad. El propósito de esta revisión es analizar la evidencia actual alrededor de los diferentes factores de riesgo asociados con la aparición de enfermedad renal crónica.

Palabras clave: Enfermedad renal crónica, factores de riesgo, mortalidad.

\section{CHRONIC RENAL DISEASE RISK FACTORS}

\section{Summary}

The chronic renal disease is a very frequent pathology associated with multiple comorbidities. The pathophysiology of the endothelial damage is different in chronic renal disease patients and this is presently a research domain. The aim of this review is to analyze the current evidence around the different risk factors associated with the appearing of chronic renal disease.

Key words: Chronic renal disease, risk factors, mortality.

\section{FATORES DE RISCO DE DOENÇA RENAL CRÔNICA}

\section{Resumo}

A doença renal crônica é uma patologia muito frequente associada com múltiplas coomorbilidades. A fisiopatologia do dano endotelial é diferente nos pacientes com doença renal crônica e este é um campo de pesquisa na atualidade. O propósito desta revisão é analisar a evidência atual ao redor dos diferentes fatores de risco associados com a aparição de doença renal crônica.

Palavras chave: Doença renal crônica, fatores de risco, mortalidade.

Correspondencia: Roberto D’Achiardi Rey M.D, rodachi@gmail.com.Dirección postal: Tr. 3 № 49-00. Facultad de Medicina, Universidad Militar Nueva Granada. Bogotá, Colombia.

Recibido: noviembre 15 de 2011 Aceptado: diciembre 28 de 2011 


\section{Introducción}

La enfermedad renal crónica (ERC) es una de las patologías de más alta prevalencia a nivel mundial. En la actualidad se estima que el 11,5\% de la población adulta en los Estados Unidos padece de ERC y esta incrementa la prevalencia de enfermedad cardiovascular $(1,2)$. Detener la progresión en el deterioro de la Filtración Glomerular (FG) se ha convertido en el objetivo terapéutico primordial para los clínicos en la actualidad.

\section{Definición y aspectos epidemiológicos de la ERC}

La ERC se define como una alteración funcional o estructural del riñón que se desarrolla de forma silenciosa, progresiva e irreversible (3, 4). Más especificamente la ERC se define como el daño renal o disminución de la FG a menos de $<60 \mathrm{ml} / \mathrm{min} / 1.73$ $\mathrm{m}^{2}$ por un período de por lo menos 3 meses.(5)

La verdadera incidencia y prevalencia de la ERC dentro de una comunidad es dificil de evaluar, ya que los estados iniciales usualmente son asintomáticos (6); sin embargo, diversas investigaciones han evaluado este aspecto. En Estados Unidos el NHANES III (National Health and Nutrition Examination Survey III) (1.988 - 1.994) estimó que cerca del $11 \%$ de la población presentaba algún grado de ERC (alrededor de 19 millones de habitantes); el 3\% de la población tenía elevación de la creatinina y el $70 \%$ de estos pacientes tenían hipertensión arterial (HTA); la prevalencia de albuminuria fue del 12\%. (6) En el Reino Unido la cohorte EPIC- Norfolk (European Prospective Investigation of Cancer and Nutrition) en 23.964 pacientes entre los 29 y los 40 años, encontró una prevalencia de microalbuminuria y macroalbuminuria del $11.8 \%$ y $0.9 \%$ respectivamente.(7) La edad, el género femenino, la presión arterial sistólica y el tabaquismo, fueron predictores independientes de la aparición de albuminuria.

En el Reino Unido, la incidencia de ERC con requerimiento de terapia de reemplazo renal crónico (TRRC) es de 100 pacientes por millón de habitantes al año. Esto es el doble respecto a los datos de la década pasada, y se espera que siga aumentando de $5 \%$ a $8 \%$ por año.(8).

En Colombia se calculó la prevalencia de ERC en TRRC en 181 ppm en el año 2001 (9) y en 450 ppm en 2007; Tomando como base las estadísticas del NHANES III, se podría estimar que en Colombia hay 4.600.00 pacientes con ERC.(10)

La elevación de la incidencia de la ERC a nivel mundial refleja en gran parte el envejecimiento progresivo de la población y la epidemia global de diabetes mellitus (DM) tipo 2. (11)

El costo del manejo de los pacientes con ERC es alto. Se esperaba que para el año 2.010 más de 2 millones de individuos en el mundo requirieran TRRC a un costo aproximado de un trillón de dólares. Muchos países en vía de desarrollo no tienen posibilidad de brindar estas terapias, por lo cual la mortalidad sigue siendo alta. (12)

\section{Factores de riesgo que afectan la progresión de la enfermedad renal crónica}

Los factores de riesgo cardiovascular que favorecen la aparición o afectan la progresión de la ERC, pueden ser modificables y no modificables.

\section{Factores de riesgo no modificables}

\section{Predisposición genética}

Múltiples estudios genéticos han sugerido relación entre la ERC y la variedad de polimorfismos de múltiples genes que sintetizan moléculas, como son los factores del eje sistema renina angiotensina aldosterona, la óxido nítrico sintetasa, el factor de necrosis tumoral alfa y múltiples citoquinas.(13)

\section{Factores raciales}

Tienen un papel muy especial en la susceptibilidad a la ERC, reflejada en la alta prevalencia de HTA y DM en la población afroamericana y en los afro-caribeños. (14) Factores socioeconómicos como la deprivación social y el estado socioeconómico bajo, se ha asociado con aumento en la prevalencia de ERC.(15)

\section{Factores materno - fetales}

La desnutrición materna durante el embarazo y el exceso de ingesta de calorías por el recién nacido, pueden favorecer la aparición de HTA, DM, síndrome metabólico y ERC en la vida adulta. El bajo peso al nacer se ha asociado con HTA por un número redu- 
cido de nefronas al nacer (oligonefronia), que por la incapacidad de manejar cantidades altas de solutos y cargas de sal, lleva a hipertrofia compensatoria, que favorece la aparición de glomeruloesclerosis y ERC.(16)

\section{Edad}

La tasa de progresión de la ERC es influenciada por el incremento progresivo de la edad (17).

\section{Género}

En análisis univariados, el género masculino se asoció con mayor deterioro de la FG (18), pero este comportamiento no se ha podido confirmar en análisis multivariados (19).

\section{Factores de riesgo modificables}

Dentro de los predictores de progresión acelerada de la ERC se han documentado en la literatura como factores de riesgo los siguientes:

\section{CONTROL DE LA PRESIÓN ARTERIAL}

El control de la presión arterial (PA) es una meta clara dentro del manejo del paciente con ERC.

La elevación de las cifras de PA elevada a nivel sistémico, se hanrelacionado con un aumento de la la presión a nivel del glomérulo,ocasionando alteraciones crónicas hemodinámicas de la arteriola aferente y llevando a un fenómeno conocido como hiperfiltración adaptativa. Esta es posiblemente la fase inicial de la ERC. Los cambios hemodinámicos de mayor relevancia en este proceso son $(20,21)$ :

i. Respuesta compensadora de la nefrona para mantener la FG.

ii. Vasodilatación renal primaria, que ocurre en los pacientes con diabetes mellitus y otros desordenes.

iii. Reducción compensatoria de la permeabilidad de la pared del capilar glomerular a pequeños solutos y agua. La caída de la FG es soportada por un aumento de la presión intraglomerular, respuesta mediada por una reducción del flujo hacia la macula densa con la subsecuente activación túbulo glomerular(22).
Es importante recalcar que no solo las patologías que comprometen el glomérulo tienen importancia en la progresión de la ERC; también encontramos patologías que comprometen el túbulo, causando lesión del mismo y una progresión acelerada de la enfermedad renal.

\section{PROTEINURIA Y ENFERMEDAD RENAL}

El control de la proteinuria es una meta terapéutica bien establecida en el paciente con ERC, como lo recomienda la American HeartAsociation. La presencia de proteinuria se ha considerado como un factor de riesgo independiente de enfermedad cardiovascular y progresión de la enfermedad renal.

Múltiples estudios y varias revisiones sistemáticas de la literatura confirman la asociación entre la proteinuria y la presentación de eventos cardiovasculares. (23) El estudio RENAAL encuentra que los pacientes con proteinuria mayor de 3 gramos presentaron progresión a ERC en el 85\% de los pacientes y enfermedad coronaria documentada en el 45\% de los pacientes (24).

Dentro de los mecanismos propuestos de lesión renal se encuentran la toxicidad mesangial, hiperplasia y sobrecarga tubular, toxicidad directa relacionada con compuestos filtrados y posteriormente reabsorbidos a nivel tubular como transferrina, hierro y albumina unida a ácidos grasos. La inducción del factor quimiotáctico atrayente proteína 1 (MPC1) y citoquinas inflamatorias $(25,26)$. El incremento marcado en la filtración de proteínas y la reabsorción proximal de las mismas causa lesión del túbulo por liberación de lisozimas dentro del intersticio. El disminuir el grado de proteinuria con medicamentos y un mejor control de la PA pueden disminuir los cambios hemodinámicos a nivel del glomérulo lo queconduce a menor lesión y finalmente a disminuirla tasa de pérdida de función renal (27).

La búsqueda de medicamentos antiproteinúricosha sido objeto de investigación; el uso de medicamentos antihipertensivos como los inhibidores de la enzima convertidora de antiotensina(IECAS), inhibidores de los receptores de angiotensina (ARAS II), inhibidores de la hidrometilglutarilCoA, han centrado la atención de los clínicos e investigadores en los últimos años. Otras molécula como tiazolidindionas e inhibidores directos de la renina han sido investigados recientemente (28). 
Los cambios patológicos de los podocitos conocidos como podocitopatías, han tomado importancia dentro de los mecanismos iniciales de proteinuria y glomeruloesclerosis, tanto primaria como secundaria.(29)

La angiotensina II se ha implicado en el compromiso túbulo intersticial por la presencia de receptores tipo 1 a nivel de podocitos y a nivel tubular induciendo fibrosis por la inducción de citoquinas proinflamatorias tipo TGF-beta y el factor de crecimiento del tejido conectivo. (30)

El compromiso del túbulo por fibrosis se evidencia en todas las formas de ERC. El compromiso tubular es un marcador pronóstico de la ERC (31).

\section{DISLIPIDEMIA Y ENFERMEDAD RENAL CRÓNICA}

Se ha reportado que el control metabólico, la hiperlipidemia y la acidosis metabólica se pueden relacionar con progresión de la ERC. El estudio SHARP proporcionó evidencia adecuada acerca de la eficacia y seguridad de disminuir los niveles de colesterol LDL en la incidencia de eventos ateroescleróticos mayores, en pacientes con ERC sin terapia de soporte renal. (32) Aunque se encontró descenso en el deterioro de la FG calculada por las formulas MDRD4 y por COCKCROFT GAULT en pacientes tratados con simvastatina, no se alcanzó diferencia significativa en términos estadísticos, , sin embargo, la estatina puede tener un efecto renoprotector en aquellos pacientes con ERC y enfermedad cardiovascular.

\section{TABAQUISMO}

El tabaco incrementa la PA y afecta la hemodinámica renal.(33). Tanto en pacientes diabéticos como en los no diabéticos, el tabaco es un factor de progresión independiente de la ERC.(34)

\section{FÓSFORO}

La acumulación de fósforo es un problema frecuente en los pacientes con ERC, el cual se inicia tan pronto como la función renal disminuye. (35)

Los niveles altos de fosforo se asocian con una progresión más acelerada de FG en el paciente ERC. Se ha encontrado un Hazard Ratio (HR) de 1,3 para doblar las cifras de creatinina plasmática, en pacientes cuya concentración de fosforo aumenta $1 \mathrm{mg} / \mathrm{dl}$ con respecto al límite superior (5,5 mg/dl).(36)

Otro mecanismo implicado en el desarrollo de fibrosis y atrofia tubular es el depósito de calcio en el intersticio renal, lo que lleva aprocesos inflamatorios crónicos llevando a fibrosis y atrofia tubular (37).

\section{NIVELES PLASMÁTICOS DE ALDOSTERONA}

Los niveles altos de aldosterona ha demostrado que pueden contribuir al deterioro de la función renal, como resultado de exceso en la estimulación del receptor mineralocorticoide (38), resultando en remodelación y aparición de fibrosis intersticial. El tratamiento con IECAS y ARAS II parece no ser adecuado, dados fenómenos como el escape de aldosterona y otras vías de activación que evitan su bloqueo completo. (39) Pocos estudios han mostrado beneficio con la administración de antagonistas de los receptores mineralocorticoides en ERC. El uso de bloqueadores parciales del receptor como la espironolactona, ha sido estudiado para disminuir la proteinuria sin embargo el desarrollo de hiperpotasemia ha limitado su uso en la práctica clínica, pero si se ha demostrado disminución en los niveles de proteinuria. (40)

\section{HIPERURICEMIA}

La elevación de los niveles de acido úrico se presenta en los pacientes con ERC, por disminución en la excreción urinaria. La hiperuricemia ha demostrado ser un factor de progresión de la enfermedad renal, en parte por disminución en la perfusión renal por estimulación en la proliferación de la musculatura en la arteriola aferente.(41) Los estudios clínicos han mostrado resultados contradictorios, sin tener la capacidad de demostrar asociación directa que las cifras elevadas de ácido úrico aceleren el deterioro de la función renal, por lo anterior,se deber examinar con detenimiento la utilización de medicamentos con el propósito del control de hiperuricemia y no emplearlos de manera rutinaria. (42)

\section{OBESIDAD}

La obesidad ha sido determinada en varios estudios como un factor de riesgo para el desarrollo de ERC y progresión de la misma. (43) 
Se ha visto en la población obesa mayor prevalencia de proteinuria, con el desarrollo de glomeruloesclerosis focal y segmentaria, como hallazgo en la histopatología renal de estos pacientes. La fisiopatología no es del todo conocida, se han propuesto teorías acerca de cambios hemodinámicos, aumento de sustancias vasoactivas, fibrogénicas, entre las que se incluyen la angiotensina II, insulina, leptina y factor de crecimiento transformante beta. (44)

Dentro de los cambios hemodinámicos reportados se ven fenómenos de hiperfiltración glomerular en los pacientes obesos, así como reabsorción de sodio tubular mayor al promedio de la población general. (45)

La hiperlipidemia es un trastorno frecuente en los pacientes obesos, como la hiperglicemia y otros trastornos metabólicos. En múltiples modelos animales de roedores, se ha encontrado la acumulación de vesículas de triglicéridos y colesterol a nivel de medula renal. Otras sustancias, comoel activador de plasminógeno 1 (PAI-1), Factor de Crecimiento Vascular Derivado del Endotelio (VEGF), Colágeno tipo IV y Fibronectina, se encuentran elevados en los pacientes obesos. (46)

La activación del Sistema Renina Angiotensina Aldosterona proveniente del tejido adiposo visceral favorece la elevación de los niveles plasmáticos de renina y Angiotensina II característicos de estos pacientes y que contribuyen a los cambios hemodinámicos y renales. Los niveles altos de aldosterona son comunes en los obesos y estos niveles de aldosterona son independientes de los niveles de renina, favoreciendo mas reabsorción de sodio a nivel de la nefrona distal. (47) En estos pacientes la hiperinsulinemia favorece la presencia de factores de crecimiento dependientes de insulina que llevan a la formación de glomeruloesclerosis. (48)

La leptina y adiponectina han mostrado correlación con eventos cardiovasculares y ERC (49). Niveles elevados de leptinase han correlacionado con aparición de proteinuria y glomeruloesclerosis en pacientes con índices de cintura mayor de $130 \mathrm{~cm}$. Los niveles de adiponectina se encuentran relativamente bajos cuando el diámetro de la cintura es mayor de $130 \mathrm{~cm}$, lo cual constituye un factor de riesgo para desarrollo de ERC y eventos cardiovasculares. (50)

\section{HIPERGLICEMIA}

En la actualidad es claro que un buen control de la glicemia, lleva a retardo en el deterioro de la función renal como lo mostro el DCCT, UKPDS $(51,52)$.

\section{ALCOHOL Y OTRAS}

Alguna evidencia soporta que el consumo de alcohol (53) de mas de 1,5 onzas líquidas (44 ml) ( whisky americano o escocés, vodka, ginebra, etc.) o 4 onzas líquidas (118 ml) de vino o 12 onzas líquidas (355 ml) de cerveza al día puede favorecer la HTA y ser factor de progresión de la ERC.(54)

\section{La ERC como factor de riesgo cardiovascular}

Estudios como el de la KayserFoundation (55) y el Valiant, realizados en población post infarto de miocardio (56) encuentran una clara asociación de la ERC como FRCV independiente. Es así como en la actualidad se recomienda la detección temprana de la ERC por medio del cálculo rutinario de la FG con formulas internacionales como el MDRD4 (57) y/o CKD-EPI o el cálculo de la depuración de creatinina por la formula de CockroftGault (58), con el fin de ser estrictos en las metas, manejo y seguimiento de estos pacientes.

\section{Conclusiones}

En resumen, hay diversos factores de riesgo cardiovascular, no modificables y modificables, que se relacionan con la génesis o deterioro de la función renal; estos últimos son susceptibles de intervención, que debemos asumir en forma estricta, para llevar las cifras de presión arterial o los diversos parámetros bioquímicos al normal exigido, para que la función renal se preserve o no se deteriore. Por otra parte, debemos conocer en forma clara la función renal calculada, para poner aún más énfasis en este grupo de pacientes, y ejercer un control aún mas estricto sobre ellos.

\section{Bibliografía}

1. Coresh J, Selvin E, Stevens LA, et al. Prevalence of chronic kidney disease in the United States. JAMA. 2007;298(17):20382047.

2. Weiner DE, Tighiouart H, Amin MG, et al. Chronic kidney disease as a risk factor for cardiovascular disease and all-cause mortality. J Am SocNephrol. 2004; 15(5):1307-1315. 
3. NKF Clinical Practice Guidelines for CKD. Am J Kidney Dis. 2002; 39: S 17- S 31.

4. Levey AS, Andreoli SP, et al. Chronic Kidney Disease: Common, harmful andtratable. World Kidney Disease Day. J Am Soc Nephrology 2007; 18: $374-378$.

5. National Kidney Foundation: K/DOQUI kidney disease outcome quality initiative. Am J Kidney Dis 2002;39: (Suppl 1): S1 - S266

6. Coresh J, Wei GL, Maquillan G, et al. Prevalence of high blood pressure and elevated serum creatinine level in the united states: Findings from the Third National Health in the United States Survey, 1.988 - 1.994. Arch Intern Med 2.001; 161: $1207-1216$.

7. McFadden E, Luben $\mathrm{R}$, et al. Occupational social class, educational level, smoking and body mass index, and causespecific mortality in men and women: a prospective study in the European Prospective Investigation of Cancer and Nutrition in Norfolk (EPIC-Norfolk) cohort.Eur J Epidemiol. 2008;23(8):511-22.

8. Levey AS, Eckardt KU, Tsukamoto Y, et al. Definition and clasification of chronic disease: a position statement from kidney disease: improving global outcome (KDIGO). KidneyInt 2.005; 67: 2089- 2100 .

9. Rivera A, et al. Enfermedad Renal Crónica. En manejo integral de la Enfermedad Renal Crónica en Colombia. Rivera A et al, eds, Primera Edición,k Editorial Alkianza Producciones, 2009;29-39.

10. Pereira BJ. Introduction: New perspectives in chronic renal insufficiency. Am J Kidney Dis2000; 36:Supp3: S1-3.
11. Hannedouche T, Chauveau P, Kalou F, et al: Factors affecting progression in advanced chronic renal failure. ClinNephrol39: $1993 ; 312-320$.

12. U.S. Renal Data System (UDRDS): 2006 Annual Data Report: Atlas of end stage renal disease in the united states. National Institutes of Health, 2.006. Avalaible at www.usdrs.org.

13. Locatelli F, Del Vecchio L: Natural history and factors affecting the progresión of chronic renal failure. In El Nahas Am, Anderson S, Harris (eds): Mechanism and Management of progressive renal failure. London, Oxford universitypress, 2000 , pp $20-79$.

14. Hannedouche T, Chauveau P, Kalou F, et al: Factors affecting progression in advanced chronic renal failure. ClinNephrol39 : 1993;312 -320.

15. Khan H, Catto GRD, Edward N, MacLeod AM. Chronic renal failure: factors influencing nephrology referral. Q J Med1994;87: 559-564.

16. Menon V, Sarnak MJ: The epidemiology of chronic kidney disease stages 1 to 4 and cardiovascular disease: a high risk combination. Am J Kidney Dis 2005; 45: 223 - 232.

17. Neugarten J, Acharya A, Silbiger SR: Effect of gender on the progression of nondiabetic renal disease: A meta-analysis. J Am Soc Nephrol11:2000; 319 -329.

18. Jungers $P$, Chauveau $P$, Descamps-Latscha B, Labrunie $M$, et al. Age and gender-related incidence of chronic renal failure in a French urban area: A prospective epidemiologic study. Nephrol Dial Transplant 11:1996; 1542 -1546.

19. Himmelfarb J. Chronic kidney disease and the public health: gaps in evidence from interventional trials. JAMA 2007;297:2630. 